\title{
FAR-INFRARED ABSORPTION DUE TO IMPURITIES IN CSBR CRYSTALS
}

\author{
W. PRETTL \\ Max-Planck-Institut für Festkörperforschung, Stuttgart, Germany \\ and \\ E. SIEP \\ Physikalisches Institut der Universität, Freiburg, Germany
}

Received 16 June 1971

The far-infrared absorption in the phonon band of $\mathrm{CsBr}$ due to $\mathrm{Na}^{1}, \mathrm{~K}^{1}, \mathrm{~Kb}^{+}, \mathrm{In}^{+}$, and ' $\mathrm{I}^{+} \mathrm{I}^{\text {impurities }}$ has been measured and compared with the calculated density of phonon states of the host lattice.

In a previous paper [1] we reported impurity induced far-infrared absorption in CsI crystals. Now we present the corresponding results for CsBr. The additional absorption due to $\mathrm{Na}^{+}, \mathrm{K}^{+}$, $\mathrm{Rb}^{+}, \mathrm{In}^{+}$and $\mathrm{Tl}^{+}$defect ions in $\mathrm{CsBr}$ has been measured in the far-infrared from $10 \mathrm{~cm}^{-1}$ up to about $70 \mathrm{~cm}^{-1}$. At higher frequencies the absorption of the host lattice strongly increased due to its fundamental vibration at $79.1 \mathrm{~cm}^{-1}$.

The $\mathrm{In}^{+}$doped sample was grown with the vacuum Bridgeman method. Its impurity concentration is only approximately known. All other crystals were grown by the Czochralsky-technique in an argon atmosphere. The concentrations of $\mathrm{Na}$ and $\mathrm{K}$ impurities were measured by flame spectroscopy. This method failed for $\mathrm{Rb}$ because its emission lines are too near to that of $\mathrm{Cs}$. Thus, the concentration of $\mathrm{Rb}^{+}$ions only in the $\mathrm{CsBr}$ melt is quantitatively known. The $\mathrm{Tl}^{+}$concentration was determined by colorimetric analysis. The experimental conditions were the same as described in ref. [1].

The impurity induced absorption measured at sample temperatures of about $6^{\circ} \mathrm{K}$ are shown in fig. 1. For the purpose of comparison the phonon density of states calculated by $\mathrm{H}$. Jex [2] using Schröder's BSM [3] is also plotted in fig. 1 and the phonon frequencies at critical points are indicated by the corresponding irreducible representation of the space group of the host crystal $\mathrm{O}_{\mathrm{h}}^{1}$

Fig. 1. Measured impurity induced absorption in $\mathrm{CsBr}$ at $6^{\circ} \mathrm{K}$ and calculated density of phonons at $4.2^{\circ} \mathrm{K}$ according to Jex [2].

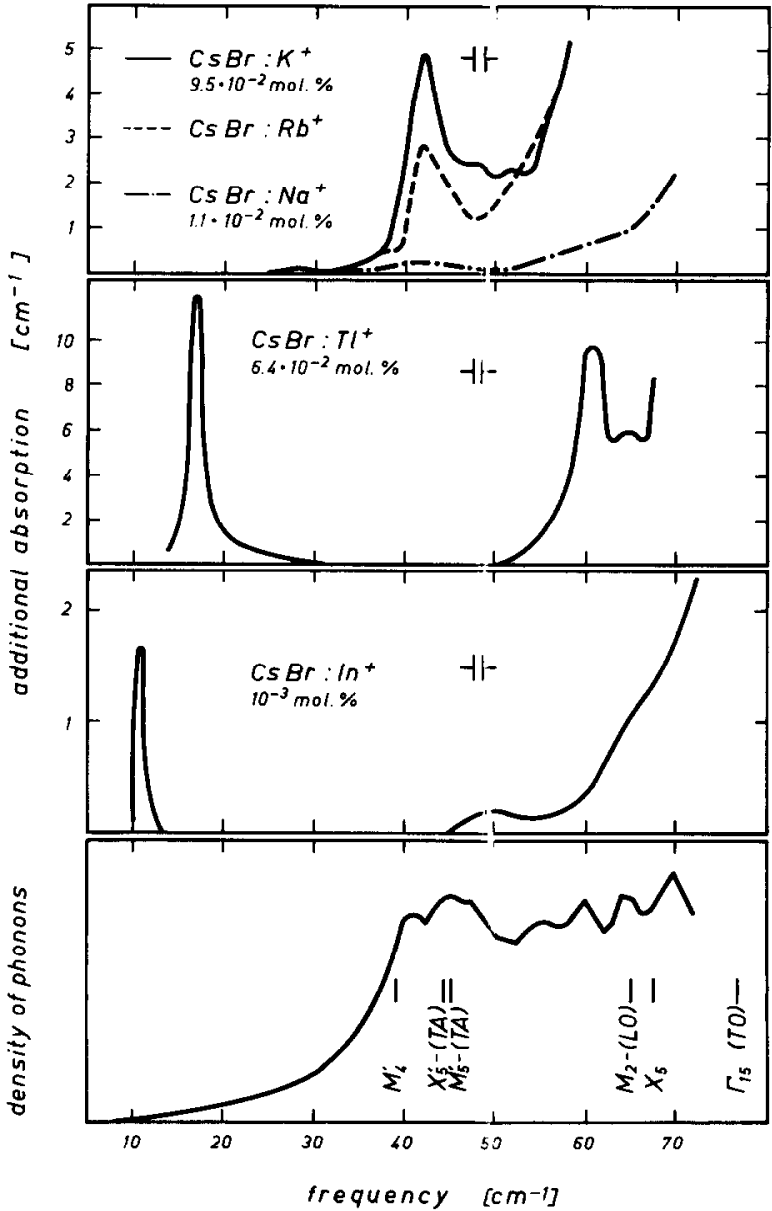


The absorption spectra of all doped $\mathrm{CsBr}$. crystals turned out to be very similar to that of CsI [1]. In the frequency range where the phonon density of states is large, all impurities cause a broad absorption. The $\mathrm{K}^{+}$and $\mathrm{Rb}^{+}$doped samples exhibit distinct absorption bands in the vicinity of $40 \mathrm{~cm}^{-1}$. This structure is also found in $\mathrm{CsBr}: \mathrm{Na}^{+}$but to a lesser extent. These bands can be due either to an activated peak in the density of states of the host crystal or to an impurity resonance. The first explanation is supported by the fact that the absorption bands occur approximately at the calculated frequencies of the phonons at critical points $M_{5}^{i}$ (TA), $M_{4}^{1}$ and $X_{5}^{\prime}$ (TA). It is just these critical points which become optically active by introducing impurities which substitute $\mathrm{Cs}^{+}$ions. On the other hand in CsI doped with $\mathrm{Na}^{+}, \mathrm{K}^{+}$and $\mathrm{Rb}^{+}$corresponding absorption maxima occurred at the same relative frequencies compared with the density of states. In a theoretical study based on the BSM, T. P. Martin showed that those bands are due to incipient impurity resonances rather than to activated peaks in the density of states [4]. Assuming this result to be valid also for $\mathrm{CsBr}$ yields some discrepancies between our measurements and the calculated density of states, because a resonance occurring at a maximum in the phonon density would be smeared out. To clarify this question a theoreti- cal investigalion using a realistic defect model is necessary.

$\mathrm{In}^{+}$and $\mathrm{Tl}^{+}$impurities induce low frequency narrow resonances the properties of which have already been discussed [5]. At higher frequencies the absorption curves do not bear any resemblance to the phonon density. This might result from the weak coupling of these impurities to the lattice which is indicated by the low frequency resonant modes.

We thank Professor Dr. L. Genzel, Dr. T. P. Martin and Dr. H. Jex for many interesting discussions. We are also indebted to Dipl. Phys. M. Schuhmacher who grew the crystals and to Dr. K. Maier, University of Regensburg, who placed the $\mathrm{In}^{+}$doped sample at our disposal. The support of the Michelson interferometer by the Deutsche Forschungsgemeinschaft is gratefully appreciated.

\section{REFERENCES}

[1] L. Genzcl, W. Prettl and E. Siep, Opt, Commun. 1 (1969) 28.

[2] H. Jex, private communication.

[3] V. Nifsslein and U. Schröder, Phys. Stat. Sol. 21 (1967) 309.

[4] T. P. Martin, J. Phys. C. , to be published.

[5] W. Prettl and E. Siep, Phys. Stat. Sol. 44b (1971) 759. 